\title{
Characteristics and outcomes of in-hospital cardiac arrest in COVID-19. A systematic review and meta-analysis
}

\author{
Lukasz Szarpak ${ }^{1,2} \odot$, Magdalena Borkowska ${ }^{2}$, Frank W. Peacock ${ }^{3}$, \\ Zubaid Rafique ${ }^{3} \odot$, Aleksandra Gasecka ${ }^{4,5} \odot$, Jacek Smereka ${ }^{6,7} \odot$, \\ Katarzyna Pytkowska ${ }^{1} \oplus$, Marta Jachowicz ${ }^{8}$, Lukasz Iskrzycki, ${ }^{6}{ }^{\oplus}$, \\ Natasza Gilis-Malinowska ${ }^{9}$, Milosz J. Jaguszewski ${ }^{9} \odot$
}

${ }^{1}$ Institute of Outcomes Research, Maria Sklodowska-Curie Medical Academy, Warsaw, Poland; ${ }^{2}$ Maria Sklodowska-Curie Białystok Oncology Center, Bialystok, Poland; ${ }^{3}$ Henry JN Taub Department of Emergency Medicine, Baylor College of Medicine, Houston, TX, United States; ${ }^{4}{ }^{\text {st }}$ Chair and Department of Cardiology, Medical University of Warsaw, Poland; ${ }^{5}$ Department of Cardiology, University Medical Center Utrecht, The Netherlands; ${ }^{6}$ Department of Emergency Medical Service, Wroclaw Medical University, Wroclaw, Poland; ${ }^{7}$ Polish Society of Disaster Medicine, Warsaw, Poland;

${ }^{8}$ Students Research Club, Maria Sklodowska-Curie Medical Academy, Warsaw, Poland;

${ }^{9} 1^{\text {st }}$ Department of Cardiology, Medical University of Gdansk, Poland

\section{This paper was guest edited by Prof. Togay Evrin}

\begin{abstract}
Background: The purpose herein, was to perform a systematic review of interventional outcome studies in patients with in-hospital cardiac arrest before and during the coronavirus disease 2019 (COVID-19) pandemic period.

Methods: A meta-analysis was performed of publications meeting the following PICOS criteria: (1) participants, patients $>18$ years of age with cardiac arrest due to any causes; (2) intervention, cardiac arrest in COVID-19 period; (3) comparison, cardiac arrest in pre-COVID-19 period; (4) outcomes, detailed information for survival; (5) study design, randomized controlled trials, quasi-randomized or observational studies comparing cardiac arrest in COVID-19 and pre-COVID-19 period for their effects in patients with cardiac arrest.

Results: Survival to hospital discharge for the pre-pandemic and pandemic period was reported in 3 studies ( $n=1432$ patients) and was similar in the pre-pandemic vs. the pandemic period, $35.6 \%$ vs. $32.1 \%$, respectively (odds ratio [OR] 1.72; 95\% confidence interval [CI] 0.81-3.65; $p=0.16$; $\left.I^{2}=72 \%\right)$. Return of spontaneous circulation was reported by all 4 studies and were also similar in the pre and during COVID-19 periods, $51.9 \%$ vs. 48.7\% (OR 1.27; 95\% CI 0.78-2.07; $p=0.33 ; I^{2}=71 \%$ ), respectively. Pooled analysis of cardiac arrest recurrence was also similar, $24.9 \%$ and $17.9 \%$ (OR 1.60; 95\% CI 0.99-2.57; $p=0.06 ; I^{2}=32 \%$ ) in the pre and during COVID-19 cohorts. Survival with Cerebral Performance Category 1 or 2 was higher in pre vs. during pandemic groups (27.3 vs. 9.1\%; OR 3.75; 95\% CI 1.26-11.20; $p=0.02$ ). Finally, overall mortality was similar in the pre vs. pandemic groups, $65.9 \%$ and $67.2 \%$, respectively (OR 0.67; 95\% CI 0.33-1.34; $p=0.25 ; I^{2}=76 \%$ ).

Conclusions: Compared to the pre-pandemic period, in hospital cardiac arrest in COVID-19 patients was numerically higher but had statistically similar outcomes. (Cardiol J 2021; 28, 4: 503-508)
\end{abstract}

Key words: coronavirus disease 2019, SARS-CoV-2, pandemic, in-hospital cardiac arrest, cardiopulmonary resuscitation, outcome

Address for correspondence: Jacek Smereka, Assoc Prof., PhD, MD, Department of Emergency Medical Service, Wroclaw Medical University, ul. Bartla 6, 52-443 Wrocław, Poland, tel: +48 601967070, e-mail: jacek.smereka@umed.wroc.pl

This article is available in open access under Creative Common Attribution-Non-Commercial-No Derivatives 4.0 International (CC BY-NC-ND 4.0) license, allowing to download articles and share them with others as long as they credit the authors and the publisher, but without permission to change them in any way or use them commercially. 


\section{Introduction}

In December 2019 the coronavirus disease (COVID-19) emerged in Wuhan, China and spread rapidly throughout the world causing a pandemic [1]. As of March 2021, there were over 126 million confirmed cases and over 2.7 million deaths worldwide [2].

Clinically, severe acute respiratory syndrome coronavirus 2 (SARS-CoV-2), the virus responsible for COVID-19, manifests with wide variability ranging from being asymptomatic to severe respiratory failure and death. An estimated $14 \%$ of patients with COVID-19 require hospitalization, with $2 \%$ requiring intensive care [3]. While coexisting conditions, such as, hypertension, diabetes, cardiovascular disease, obesity, chronic obstructive pulmonary disease and chronic renal failure have been associated with poor prognosis [4], respiratory failure is the leading cause of admission to the intensive care unit (ICU) [5]. Further, studies show that the mortality rate in the ICU can be greater than 35\% [6], and the leading cause of such high mortality is in-hospital cardiac arrest (IHCA) [7].

To understand the role of COVID-19 on outcomes of IHCA, we designed a systematic review of studies in adults during versus pre-pandemic periods of patients who suffered from cardiac arrest.

\section{Methods}

The study was performed following the recommendation Preferred Reporting Items for Systematic Reviews and Meta-Analyses (PRISMA) statement for reporting systematic reviews and meta-analyses [8]. Before starting the study, there was agreement on the analysis methods and the inclusion and exclusion criteria to be used. Because of its nature, this study was exempt from an institutional board review.

\section{Inclusion and exclusion criteria}

Studies included in this meta-analysis met the following PICOS criteria: (1) Participants: patients $>18$ years of age with cardiac arrest due to any causes, (2) Intervention: cardiac arrest in COVID-19 period, (3) Comparison: cardiac arrest in pre-COVID-19 period, (4) Outcomes: detailed information for survival, (5) Study design: randomized controlled trials, quasi-randomized or observational studies comparing cardiac arrest during and before the COVID-19 period for their effects in patients with cardiac arrest. Studies were excluded if they were reviews, case reports, confer- ence or poster abstracts, or articles not containing original data.

\section{Search strategy}

A computerized search of the Medline (PubMed), Embase, Cochrane Central Register of Controlled Trials (CENTRAL), Scopus, Web of Science was performed from inception to February $2^{\text {nd }}, 2021$. Titles and abstracts were screened by two authors independently (A.G. and M.P.). All retrieved articles were reviewed by two authors (J.S. and M.C.). Any disagreement was resolved through consensus or, if necessary, by discussion with a third author (L.S.).

The search was performed using the following terms: "cardiac arrest" OR "CA" OR "heart arrest" OR "circulation arrest" OR "cardiopulmonary resuscitation" OR "CPR" OR "in-hospital cardiac arrest" OR "IHCA" OR "return of spontaneous circulation" OR "ROSC" OR "cardiac ventric* fibrillation" OR "heart ventric* fibrillation" OR "pulseless ventric* tachycardia" OR "asysto*" OR "pulseless electrical activity" OR "PEA" AND "SARS-CoV-2" OR “COVID-19". Bibliographies of retrieved articles were manually checked for additional references to identify all eligible studies and achieve minimal publication bias. Only full articles in the English language were considered. All references were saved in an EndNote (EndNote, Inc., Philadelphia, PA, USA) library that was used to identify duplicates.

\section{Data extraction}

Information was recorded on study characteristics and demographics such as authors, publication year, inclusion and exclusion criteria, primary outcome, findings, as well as per group sample size and outcomes. Two reviewers (A.G., M.J.J.) independently abstracted data, which a third investigator (L.S.) independently verified. Authors of articles were contacted when data were missing or were reported in a format that did not allow statistical analysis. Care was taken to avoid inclusion of data from duplicate publications. In the case of suspected data discrepancies, the relevant author was contacted directly. Moreover, each reviewer performed independent data abstraction, using a standardized predefined data collection form.

\section{Quality assessment}

Two investigators (A.G. and L.S.) independently extracted individual study data and evaluated the studies for risk of bias. Any disagreements were discussed and resolved in a consensus meeting 
with the third reviewer (M.J.J.). The ROBINS-I tool (Risk of Bias in Non-randomized Studies of Interventions) was used to assess the quality of studies [9]. Robvis application was used to for visualize risk-of-bias assessments [10]. The scale has seven main domains (confounding, participant selection, classification of interventions, deviation from interventions, missing data, outcome measurement, and selection of reported results) and assigns one point for each of four judgements, which are rated as critical, serious, moderate or low (Suppl. Fig. S3 and Fig. S4).

\section{Outcomes}

The following outcomes were evaluated in the analysis, based on the consensus among content experts in our group with regard to important outcomes. The primary outcome was survival to hospital discharge, or 30 days (SHD), whichever came first. Secondary outcomes were return of spontaneous circulation (ROSC), recurrence of cardiac arrest, survival with favorable neurologic status (defined as a survival with Cerebral Performance Category [CPC] 1 or 2), and overall mortality.

\section{Statistical analysis}

Statistical analysis was performed using Review Manager software (v.5.4, Nordic Cochrane Center, The Cochrane Collaboration, Copenhagen, Denmark). The Mantel-Haenszel method was used to analyze dichotomous outcomes, and results are reported as odds ratios (ORs) with $95 \%$ confidence interval (CI). Continuous outcome differences were analyzed using an inverse variance model with a $95 \% \mathrm{CI}$, and values are reported as mean difference. When the continuous outcome was reported in a study as median, range, and interquartile range, means and standard deviations were estimated using the formula described by Hozo et al. [11].

All $\mathrm{p}$ values were two-tailed and considered significant if $<0.05$. Heterogeneity was quantified in each analysis by the tau-squared and I-squared statistics. Heterogeneity was detected with the chi-squared test with $n-1$ degree of freedom, which was expressed as $\mathrm{I}^{2}$. Values of $\mathrm{I}^{2}>50 \%$ and $>75 \%$ were considered to indicate moderate and significant heterogeneity among studies, respectively.

\section{Results}

\section{Study characteristics}

A total of 4 studies [12-15], reporting on 1609 patients ( $\mathrm{n}=788$ pre and $\mathrm{n}=821$ pandemic patients), met all inclusion/exclusion criteria (Table 1;

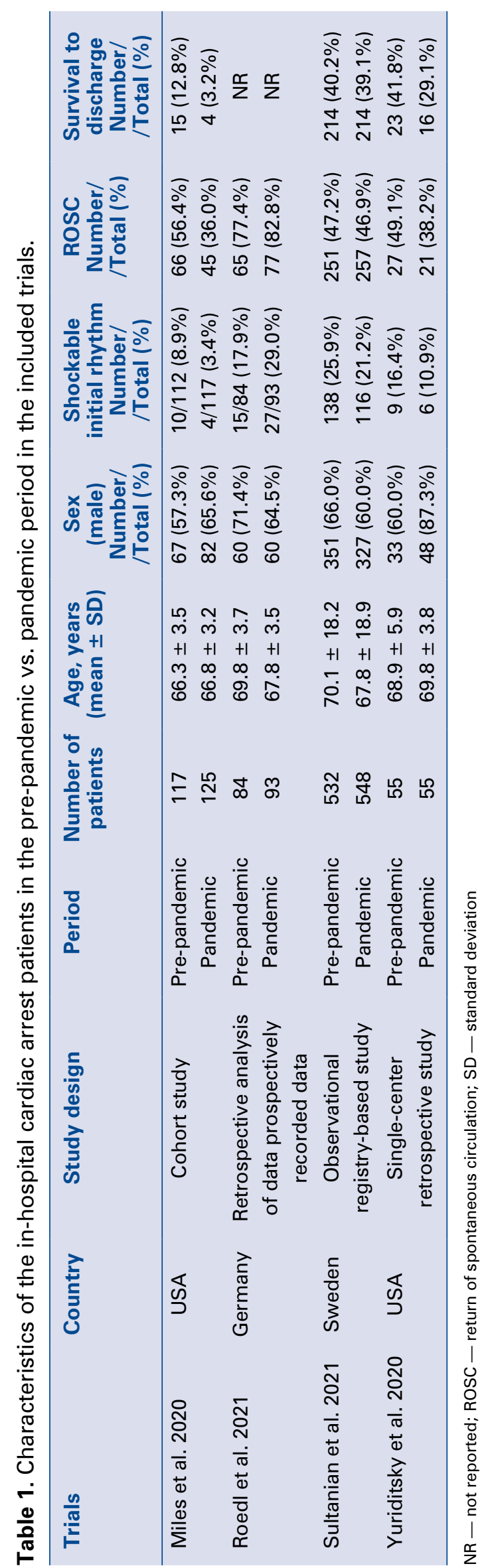




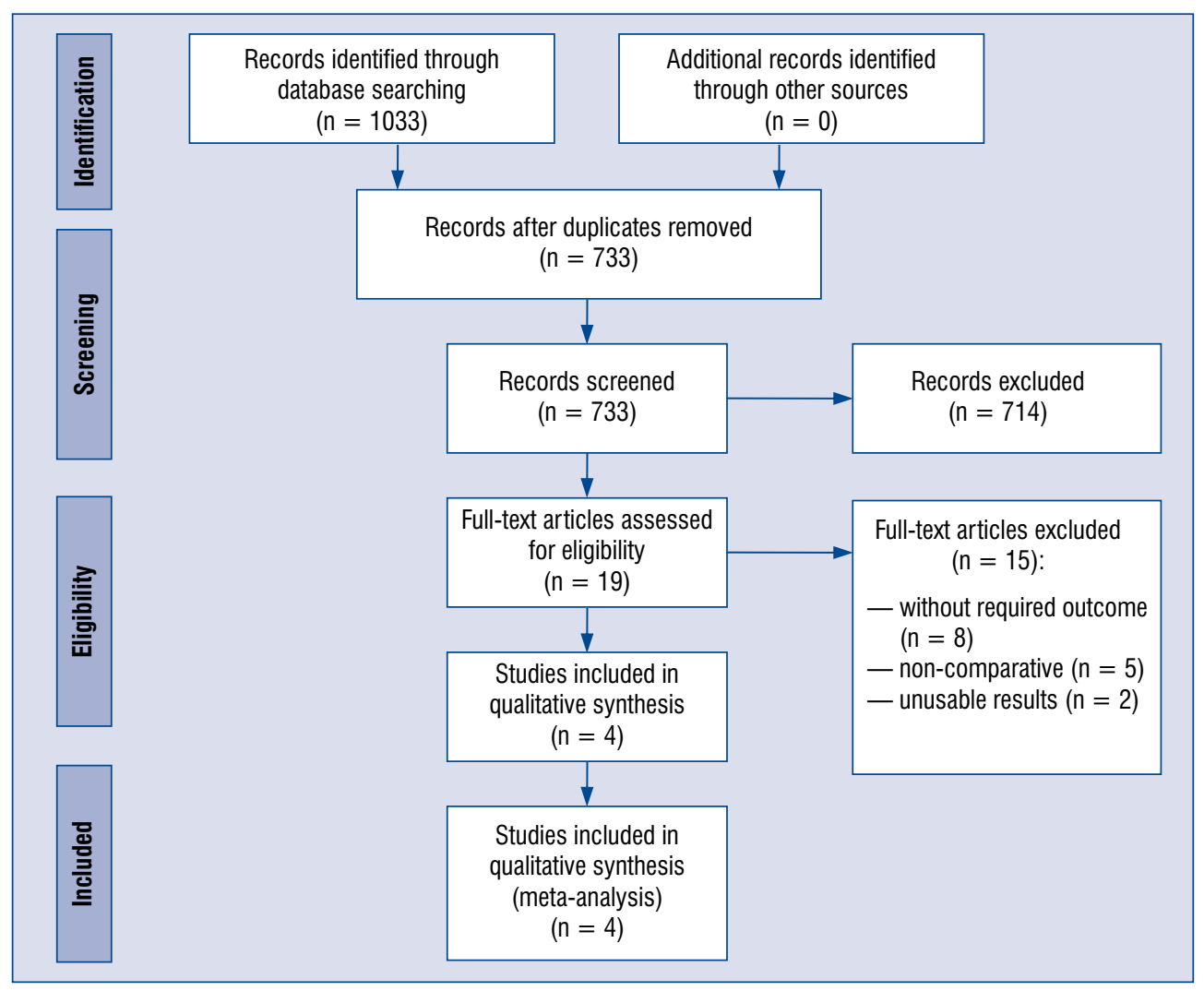

Figure 1. Flow diagram showing stages of database searching and study selection as per Preferred Reporting Items for Systematic reviews and Meta-analysis (PRISMA) guideline.

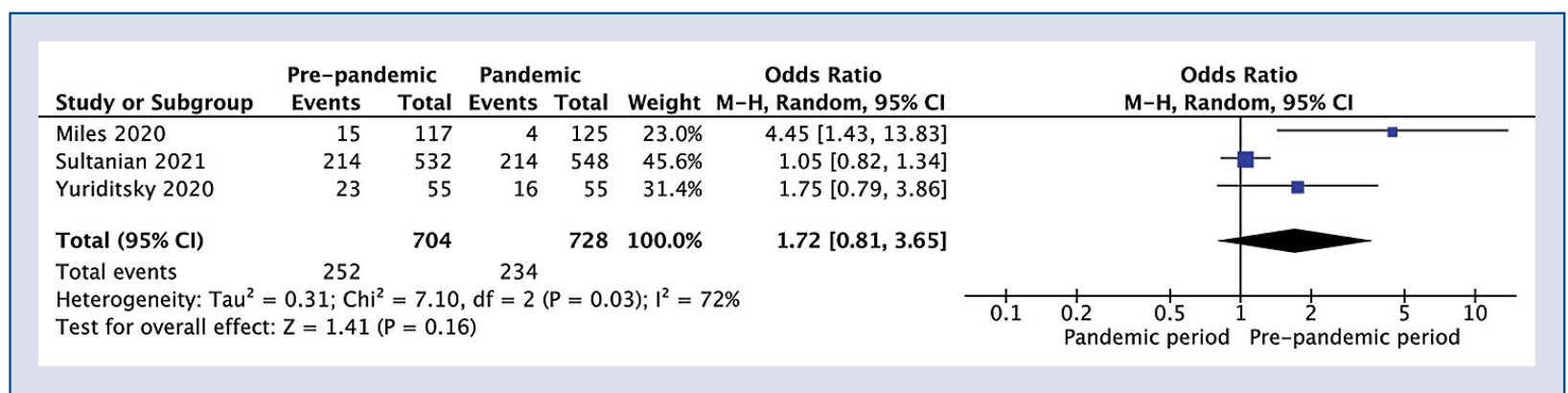

Figure 2. Forest plot of return of survival to hospital discharge between pre-pandemic versus pandemic group. The center of each square represents the weighted odds ratios for individual trials, and the corresponding horizontal line stands for a $95 \%$ confidence interval $(\mathrm{Cl})$. The diamonds represent pooled results.

Suppl. Table S1). The PRISMA flowchart (Fig. 1) summarizes the outcomes of the search strategy.

Outcomes pre-pandemic vs. pandemic period

A pooled analysis of all outcomes is presented on Figure 2 and Supplemental Figures S1 and S2. SHD for the pre-pandemic and pandemic period was reported in 3 studies ( $\mathrm{n}=1432$ patients). It occurred at a numerically higher, but a statistically similar, rate in the pre-pandemic vs. the pandemic period, $35.6 \%$ vs. $32.1 \%$, respectively (OR 1.72 ; $95 \%$ CI $\left.0.81-3.65 ; \mathrm{p}=0.16 ; \mathrm{I}^{2}=72 \%\right)$. ROSC was reported by all 4 studies, with a similar numerically higher but statistically similar relationship, $51.9 \%$ vs. $48.7 \%$ (OR $1.27 ; 95 \%$ CI $0.78-2.07$; $\mathrm{p}=0.33$; $\mathrm{I}^{2}=71 \%$ ), for the pre and during COVID-19 periods, respectively (Supplementary digital file). Pooled analysis of cardiac found an arrest recurrence of 
$24.9 \%$ and $17.9 \%$ (OR 1.60; 95\% CI $0.99-2.57$; $\left.\mathrm{p}=0.06 ; \mathrm{I}^{2}=32 \%\right)$ in the pre and during COVID-19 cohorts, respectively. Survival with CPC 1 or 2 was reported only by Yuriditsky et al. [15] and was significantly higher in pre-pandemic group compared with pandemic group (27.3 vs. $9.1 \%$; OR 3.75 ; 95\% CI 1.26-11.20; $\mathrm{p}=0.02$ ). Overall mortality in pre-pandemic vs. pandemic group was $65.9 \%$ and $67.2 \%$, respectively (OR 0.67 ; 95\% CI $0.33-1.34$; $\left.\mathrm{p}=0.25 ; \mathrm{I}^{2}=76 \%\right)$.

\section{Outcomes in pandemic period}

Only 1 study was found, Sultanian et al. [14], that compared IHCA outcomes in pandemic period between COVID-19 and non-COVID-19 patients. In this study SHD between COVID-19 and non-COVID-19 patients was $25.0 \%$ vs. $41.8 \%$ (OR $0.46 ; 95 \%$ CI $0.26-0.83 ; \mathrm{p}=0.01$ ). In the case of COVID-19 patients there was a significantly lower ROSC rate compared with non-COVID-19 patients (30.5\% vs. $52.6 \%$, respectively; OR $0.40 ; 95 \%$ CI $0.23-0.69 ; \mathrm{p}=0.001)$. Ultimately, the overall mortality in the COVID-19 patients was $75.0 \%$ and was significantly higher than that in non-COVID-19 patients $-59.3 \%$ (OR 2.06; 95\% CI 1.15-3.69; $\mathrm{p}=0.02$ ). Finally, overall mortality in the present analysis was similar in the pre vs. pandemic groups, $65.9 \%$ and $67.2 \%$, respectively (OR $0.67 ; 95 \%$ CI $\left.0.33-1.34 ; \mathrm{p}=0.25 ; \mathrm{I}^{2}=76 \%\right)$.

\section{Discussion}

According to available research, this is the first meta-analysis comparing characteristics and outcomes of IHCA before and during the COVID-19 pandemic. The primary outcome of the study was to measure the survival to hospital discharge or 30 days, whichever came first, although it was expected that mortality rates would be higher during the pandemic, it was found that the survival rates were similar to the pre-pandemic period. There are possible explanations of this phenomenon. During the pre-pandemic time the vast majority of the IHCA happened in the ICU [16], commonly the result of complications from cardiovascular disease, while during the pandemic a shift of IHCA occurring in the standard ward as a result of the pulmonary inflammatory state and fibrosis of the lung tissue [14] was observed [13].

Sustained ROSC was found, defined as stable circulation for at least $20 \mathrm{~min}$ [13] was similar in the pre-pandemic period. Interestingly in the cohort by Miles et al. [12] the pre-pandemic group was characterized by much higher ROSC. Although
ROSC is generally a positive predictor of survival [17], Sheth et al. [18] presented data which showed that although all patients with COVID-19 who suffered cardiac arrest and achieved ROSC did not achieve SHD.

Consistent with the current primary outcome, a insignificant trend was found $(\mathrm{p}=0.06)$ of lower recurrence of cardiac arrest in the pandemic group. This finding is also likely the result of the different etiology of cardiac arrests in the pre and during pandemic periods. In the pre pandemic period the vast majority of IHCA was caused by the underlying cardiac diseases [19] as opposed to the respiratory background in the COVID-19 era.

In his paper Yuriditsky et al. [15] assessed the functional status using the $\mathrm{CPC}$. This score ranges from 1 (good cerebral performance) to 5 (brain death), with CPC 1 and 2 generally categorized as good neurological outcome and 3 to 5 as poor [20]. It was reported that the pre pandemic group had a statistically significant higher survival with CPC 1 or 2 amounting to $27.3 \%$ vs. $9.1 \%$ in the COVID-19 cohort. A possible explanation may lie in the location of IHCA resuscitation. In the pre-pandemic period, many resuscitations occurred in an ICU, where early identification and greater resources may result in better outcomes than general ward resuscitations that are found later and may have fewer resources.

When comparing COVID vs. non-COVID, there was lower SHD in COVID patients, $25.0 \%$ vs. $41.8 \%$, respectively [14]. This may be because COVID patients were less likely to have a cardiac cause of the arrest, and a respiratory cause was more likely. Such a high discrepancy might be the result of the burden of critical illness with a higher likelihood or requiring ICU admission, invasive mechanical ventilation, vasopresors or renal replacement therapies than the non-COVID-19 cohort [21, 22].

Sultanian et al. [14] also reports statistically significantly lower ROSC rate for COVID-19 positive cohort with only $7.6 \%$ of patients presenting with the shockable rhythm. Overall mortality among the COVID-19 patients was $75.0 \%$ and was significantly higher than the $59.3 \%$ that occurred in non-COVID-19 patients.

\section{Limitations of the study}

Although a limitation of the present study was the strict inclusion criteria, which allowed for the inclusion of only 4 papers, it did insure a high quality of results in over 1600 patients. A second limitation is the focus on general epidemiologic 
findings, which does not allow for the pinpointing of the specific risk factors that could be used as a guideline for the selection of high-risk patients.

\section{Conclusions}

It was found that the IHCA before and during the COVID-19 pandemic had numerically higher but statistically similar outcomes.

\section{Acknowledgements}

The study was supported by the ERC Research Net and by the Polish Society of Disaster Medicine.

\section{Conflict of interest: None declared}

\section{References}

1. Rodriguez-Morales AJ, Bonilla-Aldana DK, Balbin-Ramon GJ, et al. History is repeating itself: probable zoonotic spillover as the cause of the 2019 novel coronavirus epidemic. Infez Med. 2020; 28(1): 3-5, indexed in Pubmed: 32009128.

2. Dong E, Du H, Gardner L. An interactive web-based dashboard to track COVID-19 in real time. Lancet Infect Dis. 2020; 20(5): 533-534, doi: 10.1016/S1473-3099(20)30120-1, indexed in Pubmed: 32087114 .

3. Stokes EK, Zambrano LD, Anderson KN, et al. Coronavirus Disease 2019 Case Surveillance - United States, January 22-May 30, 2020. MMWR Morb Mortal Wkly Rep. 2020; 69(24): 759-765, doi: 10.15585/mmwr.mm6924e2, indexed in Pubmed: 32555134.

4. Yang X, Yu Y, Xu J, et al. Clinical course and outcomes of critically ill patients with SARS-CoV-2 pneumonia in Wuhan, China: a single-centered, retrospective, observational study. Lancet Respir Med. 2020; 8(5): 475-481, doi: 10.1016/S2213-2600(20)30079-5, indexed in Pubmed: 32105632.

5. Ayaz A, Arshad A, Malik H, et al. Risk factors for intensive care unit admission and mortality in hospitalized COVID-19 patients. Acute Crit Care. 2020; 35(4): 249-254, doi: 10.4266/ acc.2020.00381, indexed in Pubmed: 33172229.

6. Armstrong RA, Kane AD, Kursumovic E, et al. Mortality in patients admitted to intensive care with COVID-19: an updated systematic review and meta-analysis of observational studies. Anaesthesia. 2021; 76(4): 537-548, doi: 10.1111/anae.15425, indexed in Pubmed: 33525063.

7. Shao F, Xu S, Ma X, et al. In-hospital cardiac arrest outcomes among patients with COVID-19 pneumonia in Wuhan, China. Resuscitation. 2020; 151: 18-23, doi: 10.1016/j.resuscitation.2020. 04.005, indexed in Pubmed: 32283117.

8. Moher D, Liberati A, Tetzlaff J, et al. Preferred Reporting Items for Systematic Reviews and Meta-Analyses: The PRISMA Statement. PLoS Med. 2009; 6(7): e1000097, doi: 10.1371/journal. pmed.1000097.

9. Sterne JAc, Hernán MA, Reeves BC, et al. ROBINS-I: a tool for assessing risk of bias in non-randomised studies of interventions. BMJ. 2016; 355: i4919, doi: 10.1136/bmj.i4919, indexed in Pubmed: 27733354.

10. McGuinness LA, Higgins JPT. Risk-of-bias VISualization (robvis): An R package and Shiny web app for visualizing risk-of- bias assessments. Res Synth Methods. 2021; 12(1): 55-61, doi: 10.1002/jrsm.1411, indexed in Pubmed: 32336025.

11. Hozo SP, Djulbegovic B, Hozo I. Estimating the mean and variance from the median, range, and the size of a sample. BMC Med Res Methodol. 2005; 5: 13, doi: 10.1186/1471-2288-5-13, indexed in Pubmed: 15840177.

12. Miles JA, Mejia M, Rios S, et al. Characteristics and outcomes of in-hospital cardiac arrest events during the COVID-19 pandemic: a single-center experience from a new york city public hospital. Circ Cardiovasc Qual Outcomes. 2020; 13(11): e007303, doi: 10.1161/CIRCOUTCOMES.120.007303, indexed in Pubmed: 32975134.

13. Roedl K, Söffker G, Fischer D, et al. Effects of COVID-19 on in-hospital cardiac arrest: incidence, causes, and outcome a retrospective cohort study. Scand J Trauma Resusc Emerg Med. 2021; 29(1): 30, doi: 10.1186/s13049-021-00846-w, indexed in Pubmed: 33557923.

14. Sultanian P, Lundgren P, Strömsöe A, et al. Cardiac arrest in COVID-19: characteristics and outcomes of in- and out-of-hospital cardiac arrest. A report from the swedish registry for cardiopulmonary resuscitation. Eur Heart J. 2021; 42(11): 1094-1106, doi: 10.1093/eurheartj/ehaa1067, indexed in Pubmed: 33543259.

15. Yuriditsky E, Mitchell OJL, Brosnahan SB, et al. Clinical characteristics and outcomes of in-hospital cardiac arrest among patients with and without COVID-19. Resusc Plus. 2020; 4: 100054, doi: 10.1016/.j.resplu.2020.100054, indexed in Pubmed: 33403368.

16. Perman SM, Stanton E, Soar J, et al. American Heart Association's Get With the Guidelines ${ }^{\circledR}$ - Resuscitation (formerly the National Registry of Cardiopulmonary Resuscitation) Investigators. Location of In-Hospital Cardiac Arrest in the United StatesVariability in Event Rate and Outcomes. J Am Heart Assoc. 2016; 5(10): e003638, doi: 10.1161/JAHA.116.003638, indexed in Pubmed: 27688235.

17. Wampler DA, Collett L, Manifold CA, et al. Cardiac arrest survival is rare without prehospital return of spontaneous circulation. Prehosp Emerg Care. 2012; 16(4): 451-455, doi: 10.3109/10903127.2012.695435, indexed in Pubmed: 22834854.

18. Sheth V, Chishti I, Rothman A, et al. Outcomes of in-hospital cardiac arrest in patients with COVID-19 in New York City. Resuscitation. 2020; 155: 3-5, doi: 10.1016/j.resuscitation.2020.07.011, indexed in Pubmed: 32707146.

19. Moskowitz A, Holmberg MJ, Donnino MW, et al. In-hospital cardiac arrest: are we overlooking a key distinction? Curr Opin Crit Care. 2018; 24(3): 151-157, doi: 10.1097/MCC.0000000 000000505, indexed in Pubmed: 29688939.

20. Cummins RO, Chamberlain DA, Abramson NS, et al. Recommended guidelines for uniform reporting of data from out-ofhospital cardiac arrest: the Utstein Style. A statement for health professionals from a task force of the American Heart Association, the European Resuscitation Council, the Heart and Stroke Foundation of Canada, and the Australian Resuscitation Council. Circulation. 1991; 84(2): 960-975, doi: 10.1161/01.cir.84.2.960, indexed in Pubmed: 1860248.

21. Dzieciatkowski T, Szarpak L, Filipiak KJ, et al. COVID-19 challenge for modern medicine. Cardiol J. 2020; 27(2): 175-183, doi: 10.5603/CJ.a2020.0055, indexed in Pubmed: 32286679.

22. Smereka J, Szarpak L, Gadalla F, et al. Ethical and organizational dilemmas related to the treatment of COVID-19 patients. Disaster Emerg Med J. 2020, doi: 10.5603/demj.a2020.0030. 